\title{
What are the barriers, facilitators and interventions targeting help-seeking behaviours for common mental health problems in adolescents? A systematic review
}

Antonia Aguirre Velasco ${ }^{1}$, Ignacio Silva Santa Cruz ${ }^{2}$, Jo Billings ${ }^{3}$, Magdalena Jimenez ${ }^{4}$ and Sarah Rowe ${ }^{3 *}$ (i)

\begin{abstract}
Background: Increasing rates of mental health problems among adolescents are of concern. Teens who are most in need of mental health attention are reluctant to seek help. A better understanding of the help-seeking in this population is needed to overcome this gap.

Methods: Five databases were searched to identify the principal barriers, facilitators and interventions targeting help-seeking for common mental health problems in adolescents aged 10-19years. The search was performed in June 2018 and updated in April 2019. Two independent screening processes were made using the eligibility criteria. Quality assessment of each study was performed, and findings summarised using a narrative synthesis.

Results: Ninety studies meet the inclusion criteria for this review for barrier and facilitators $(n=54)$ and interventions $(n=36)$. Stigma and negative beliefs towards mental health services and professionals were the most cited barriers. Facilitators included previous positive experience with health services and mental health literacy. Most interventions were based on psychoeducation, which focused on general mental health knowledge, suicide and self-harm, stigma and depression. Other types of interventions included the use of multimedia and online tools, peer training and outreach initiatives. Overall, the quality of studies was low to medium and there was no general agreement regarding help-seeking definition and measurements.

Conclusion: Most of the interventions took place in an educational setting however, it is important to consider adolescents outside the educational system. Encouraging help-seeking should come with the increased availability of mental health support for all adolescents in need, but this is still a major challenge for Child and Adolescent Mental Health Services. There is also a need to develop shared definitions, theoretical frameworks and higher methodological standards in research regarding help-seeking behaviours in adolescents. This will allow more consistency and generalisability of findings, improving the development of help-seeking interventions and ensuring timely access to mental health treatments.
\end{abstract}

Keywords: Help-seeking, Adolescent, Mental health, Barriers, Facilitators, Interventions, Systematic review

\footnotetext{
* Correspondence: s.rowe@ucl.ac.uk

${ }^{3}$ División of Psychiatry, University College London, London, UK

Full list of author information is available at the end of the article
}

(c) The Author(s). 2020 Open Access This article is licensed under a Creative Commons Attribution 4.0 International License, which permits use, sharing, adaptation, distribution and reproduction in any medium or format, as long as you give appropriate credit to the original author(s) and the source, provide a link to the Creative Commons licence, and indicate if changes were made. The images or other third party material in this article are included in the article's Creative Commons licence, unless indicated otherwise in a credit line to the material. If material is not included in the article's Creative Commons licence and your intended use is not permitted by statutory regulation or exceeds the permitted use, you will need to obtain permission directly from the copyright holder. To view a copy of this licence, visit http://creativecommons.org/licenses/by/4.0/ The Creative Commons Public Domain Dedication waiver (http://creativecommons.org/publicdomain/zero/1.0/) applies to the data made available in this article, unless otherwise stated in a credit line to the data. 


\section{Background}

Young people present with the highest prevalence of mental health disorders compared to individuals at any other stage of the lifecycle [1], with up to $20 \%$ of adolescents likely to experience mental health disorders [2]. Mental health has been defined as "a state of wellbeing in which and individual realizes of his/her abilities, can cope with normal stresses of life ( ...) and is able to make a contribution to his/her community" [3]. Around $50 \%$ of mental health conditions start before the age of 14. [4] and the onset of $75 \%$ of cases is before the age of 18 [5]. The most common diagnoses are depression and anxiety [6] and around 25\% of young people experience psychological distress [7]. Depression is one of the principal causes of illness and disability in teenagers, and suicide is the third most common cause of death among older adolescents [4]. Mental health problems can significantly affect the development of children and young people [4] having an enduring impact on their health and social functioning in adulthood [8]. Adolescents experiencing mental health conditions may face several challenges such as isolation, stigma, discrimination and difficulty in accessing health services [2]. However, 75\% of adolescents with mental health problems are not in contact with mental health services [9], the primary reason being reluctance to seek help $[1,10,11]$.

Help-seeking for mental health problems necessitates communicating the need for personal and psychological assistance to obtain advice and support. Rickwood and Thomas' (2012) define help-seeking for mental health problems as "an adaptive coping process that is the attempt to obtain external assistance to deal with mental health concerns" [p.180, 12]. This includes both formal (e.g., health services) and informal (e.g., friends and family) sources of help. However, adolescents most in need of psychological help are those least likely to look for it [1-13]. One of the biggest challenges in adolescent mental health is ensuring that at-risk individuals are linked with the appropriate support [14]. Understanding barriers and facilitators to help- seeking is fundamental for the development of interventions and programmes to support adolescents with mental health problems.

Rickwood et al., (2005), investigated the main barriers and facilitators of help-seeking for mental health problems in young people. They found that lack of emotional competence, negative beliefs about help-seeking and stigma were the most prominent barriers. Conversely, emotional competence, previous positive experiences with health professionals and mental health literacy, were the main facilitators [15]. Gulliver et al., (2010) performed a systematic review of the available literature at that time, finding similar results; however, they stated that stigma was the most prominent barrier for seeking for help in young people [1]. Another systematic review was made by Rowe et al., (2014), focused on in helpseeking for adolescent self-harm. They found that in addition to stigma, negative reactions from others related to confidentiality breaches and being seen as an "attention seeker" were the most relevant obstacles [10]. While interesting, these previous reviews do not address the help-seeking barriers and facilitators of most common mental health troubles among adolescents, nor include interventions targeting these. Rickwood, Deane et al., (2005) only included depressive symptoms, personal emotional problems and suicidal thoughts and Rowe et al. (2014), only focused on adolescent self-harm. The most complete review published by Gulliver and colleagues (2010) is almost 10 years old and need of updating.

Adequate and effective interventions that promote help-seeking are necessary for enhancing prevention, early detection, timely treatment and recovery from mental health problems [14]. Previous systematic reviews on interventions targeting help-seeking reveal some promising results in regard to enhancing mental health literacy [16] and a significant positive overall effect of these interventions in improving help-seeking for mental health problems [17]. Nonetheless, these reviews do not focus on adolescent populations and only one includes randomised controlled trials (RCT).

The primary aim of this review is therefore to provide an update of the literature on barriers and facilitators of adolescent mental health help-seeking including formal and informal sources of help, with the inclusion of interventions targeted at improving this. We will focus on common mental health problems, including depression, anxiety, suicidal thoughts, self-harm, emotional distress, among other personal-emotional symptoms. The secondary outcome is to examine any significant differences between age and sex. Understanding the difficulties around help-seeking behaviours and facilitating access to timely and effective treatment is essential for preventing the escalation of mental health problems among adolescents.

\section{Methods}

For the purpose of this review, help-seeking was defined as the action of actively searching for help for mental health problems, including informal (family, friends) or formal (GP, mental health professionals, etc.) sources, based on interpersonal and social abilities [11]. "Adolescents" were people aged 10 to 19 years, as defined by the World Health Organisation [4]. Despite the increasing debate regarding the age of adolescence [18], this definition was considered as appropriate for our study as it is accepted by international organisation such as OMS and UNICEF. Also, we considered this age range more homogenous and comparable in terms of lifecycle 
experiences and challenges that would be reflected in help-seeking behaviours and intentions. This review was prospectively registered on PROSPERO (CRD42018096917) and reported in accordance with the PRISMA guidelines [19]. The search terms were developed using the PICO structure, then expanded using $\mathrm{MeSH}$ terms and combined using Boolean operators. Four databases were selected including MEDLINE', Embase, PsycINFO, and Web of Science, as well as the search engine Google scholar, identified as an optimal database combination [20]. Grey literature from the mentioned databases was also included and a search was carried in Open Grey. An initial version of the proposal for this study was reviewed by the McPin Foundation. The feedback was considered in the developmental stage, in order to evaluate the relevance and reception of the protocol by Patient and Public Involvement (PPI) organisations.

We included studies published in English, Spanish and French and focused on identifying barriers, facilitators and interventions targeting help-seeking behaviours for mental health problems in adolescents, specifically depression, anxiety, suicidal ideation, emotional distress and general symptoms of mental illness. Other mental health problems such as psychosis, anorexia, among others were excluded, because we decided to focus on most prevalent mental health problems which share a more similar help-seeking process. Regarding barriers and facilitators, we included studies published after 2010 since a previous systematic review on the topic was published then [1]. We did not include any limit regarding year of publication for help-seeking interventions. All study designs were considered, including feasibility studies and study protocols. We excluded studies that referred to young people over the age of 19 or children under 10 years old. When study populations included adolescents outside of the established age range, the paper was included if over $50 \%$ of the individuals in the sample were within the 10-19 years category or if separate outcome data was provided for the participants in this age range. Studies meeting the inclusion criteria and including parents in their sample were also considered. Finally, other exclusion criteria were articles written in other languages, or if the intervention did not explicitly target help-seeking behaviours or was not related to mental health conditions (Appendix S1) (Table 1).

The search was performed in June 2018 and updated in April 2019. The results were exported to EndNote X8 and duplicates were removed. Titles and abstracts were screened by one author (AA) at the first stage. At a second stage, two authors (AA and IS) checked the full articles using the pre-determined inclusion and exclusion criteria. A third member of the research team (MJ) was available to solve discrepancies. Disagreement on 12 studies was attributed mainly to differences concerning the definition and measurement of help-seeking and was resolved in a discussion with a third author (MJ) not involved in the process of screening. Authors were contacted when relevant information was missing or when we could not find the articles retrieved by the databases. Reference list of all included studies were screened in case we found other studies relevant to our review. Data were extracted using a predefined form, which allowed the research team to identify the main characteristics of each study. This process was executed by one author (AA) after a complete review of the included papers. For the first question, data extraction focused on identifying barriers and facilitators and for the second question, intervention and effect size when reported. We created an additional form to extract data regarding the secondary outcome (age and sex). For the quality assessment, we used the Joanna Briggs Institute Critical Appraisal Checklist [21] and the Mixed Methods Appraisal Tool (MMAT) [22], which were appropriate due to the variety of study designs included in this review; both have been previously validated [23, 24]. The Joanna Briggs tool has a number of checklists to evaluate the main features of each study design. We used the checklist for crosssectional studies, RCT, quasi-experimental studies and qualitative studies. Each checklist had a number of items to evaluate the most relevant aspect of the specific design (e.g: for RCT was allocation to treatment groups concealed? Were treatment groups similar at the baseline?). After completing the checklist an overall quality appraisal score was calculated to provide a measure (low, medium and high) of the quality of each study. The MMAT included a similar checklist but is specific to mixed method study reviews. Overall study quality was not used as an exclusion criterion because we opted to be overly inclusive and provide a thorough overview of help-seeking in adolescents. Results have been summarised using a narrative synthesis. We identified the most relevant features regarding help-seeking barriers, facilitators and interventions in our data. These features were grouped into themes that capture the essential aspects regarding the main outcome of this review. With this information we developed a preliminary synthesis of the results organizing the themes so that patterns regarding the main barriers, facilitators and interventions were identified. Finally, we explored previous evidence on the topic and explore the relationship between the included studies. This allowed us to explore the influence of heterogeneity and the robustness of the preliminary synthesis [25]. Due to the heterogeneous nature of the studies included, a meta-analysis was not conducted. The quality of each study was not used as an exclusion criterion or impacted the weight given to each study in the narrative synthesis. This over-inclusive criteria 
Table 1 Inclusion/Exlusion criteria

\begin{tabular}{ll}
\hline Inclusion & Exclusion \\
\hline - Focused on help-seeking barriers, facilitators and & - Not focused on help-seeking for mental health problems \\
interventions & - Help-seeking for anorexia, schizophrenia and other mental health problems not de- \\
- Includes formal and informal sources of help & scribed in the inclusion criteria \\
- Help-seeking for depression, anxiety, suicidal ideation, & - Participants above or over 10 to 19years age range \\
emotional distress & - Published in other languages \\
- Age range: 10 to 19years & - Published before 2010 if referring to help-seeking barriers and facilitators \\
- Studies published in English Spanish and French & \\
- All study designs & \\
\hline
\end{tabular}

allowed us to have an overall picture not only regarding help-seeking barriers, facilitators and interventions, but also the methodological quality of the available evidence.

\section{Results}

Two independent searches were carried out during June 2018 and then updated in April 2019. A total of 90 studies were included in this review, combining both barriers and facilitators $(n=54)$ and the intervention $(n=36)$ questions. PRISMA diagrams displaying the number of papers retrieved and the process of selection of the included studies is available in Figs. 1 and 2. Regarding the inter-rater reliability for this review, the agreement between the researchers screening the papers

\section{PRISMA 2009 Flow Diagram}

Question 1: Help-seeking barriers and facilitators
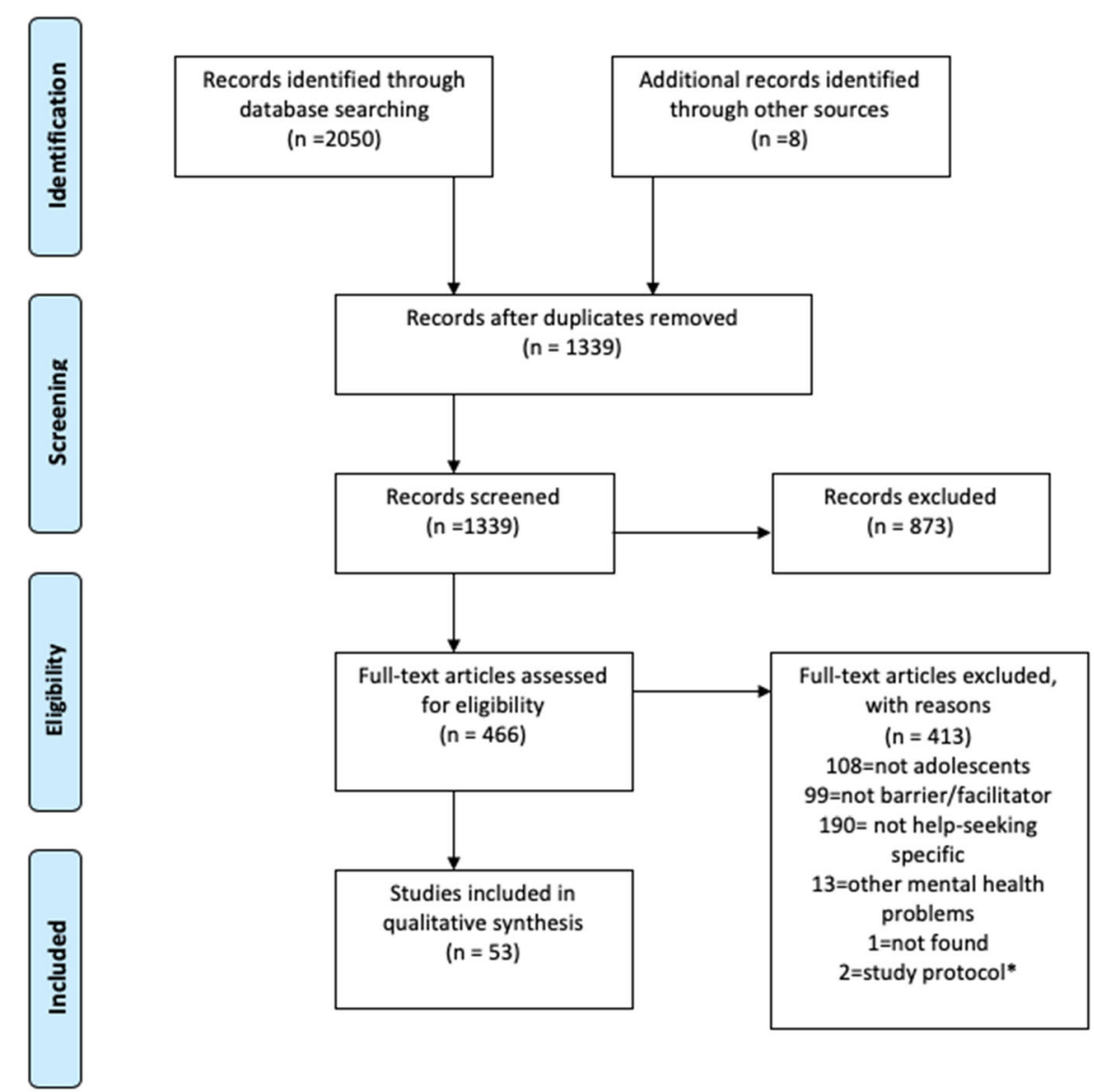

Fig. 1 Prisma 2009 Flow Diagram. Question 1: Help-seeking barriers and facilitators 


\section{PRISMA 2009 Flow Diagram}

Question 2: Help-seeking interventions

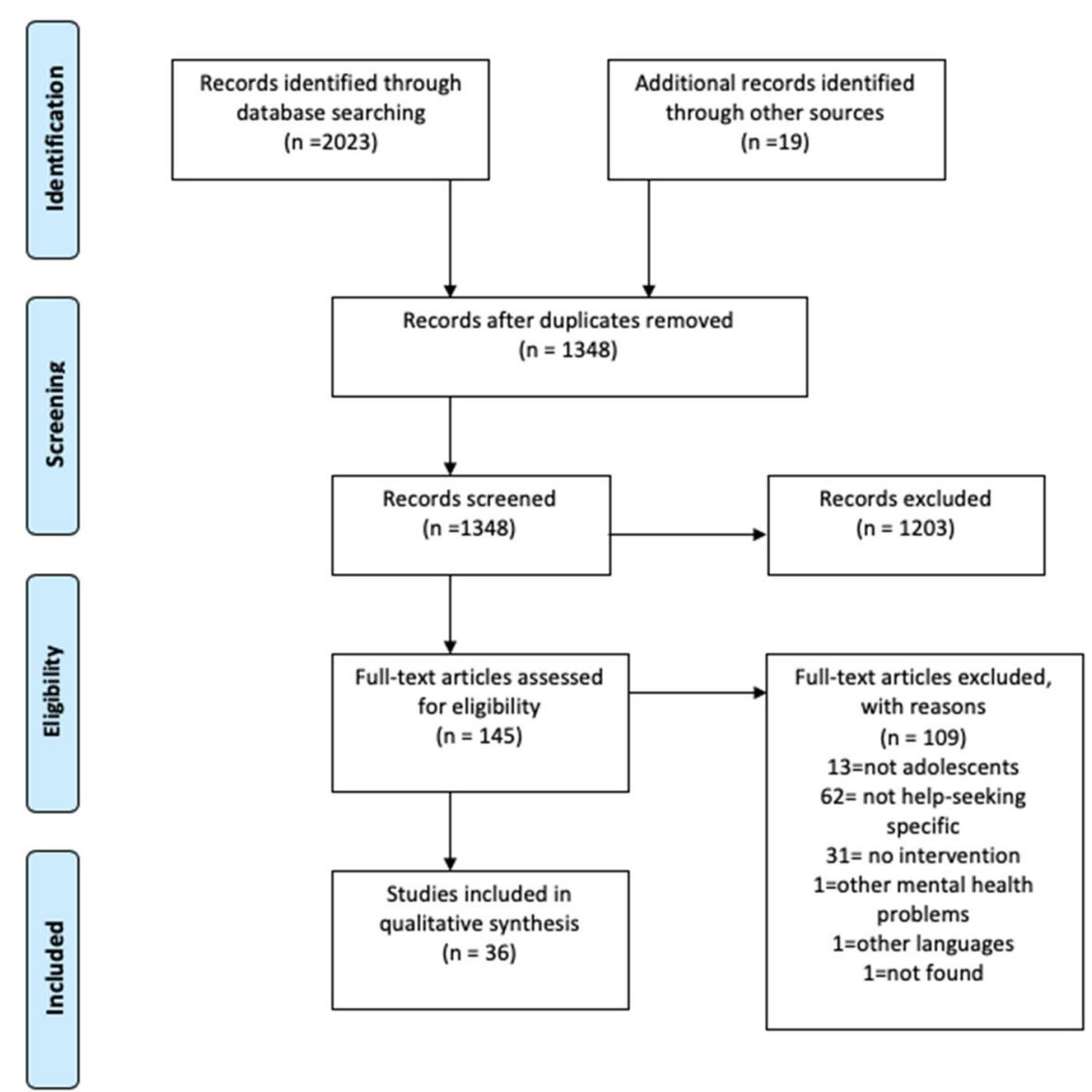

Fig. 2 Prisma 2009 Flow Diagram. Question 2: Help seeking interventions

was high, with a 85\% accuracy and 95\% precision $($ Kappa $=0.954)$. Adolescents identified a range of formal and informal help-seeking options across studies, such as GPS, psychologists, psychiatrists, teachers, social workers (formal), and friends, family, sporting coaches, and online communities (informal). Regarding question 1 , most of studies focused on identifying barriers and facilitators towards formal sources of help, whereas intervention studies had a wider variety of sources of help, depending on help-seeking behavior attempted to promote.

\section{Question 1: help-seeking barriers and facilitators}

Fifty-four studies that reported barriers and/or facilitators including a total of 56,821 participants were considered in the narrative synthesis (Table 2). Most of the studies $(n=$ 18) were conducted in Australia, followed by the United
States $(n=12)$ and the United Kingdom $(n=5)$. The majority of the studies were cross sectional $(n=36)$ [26-61], thirteen studies were qualitative [62-74] and six used a mixed-method design [75-80]. Three $\mathrm{PhD}$ dissertations and one conference abstract were included in the grey literature. The age ranged from 8 to 26 years old. Three articles included adolescents and their parents, while one article included just adolescents' mothers.

The majority of studies were conducted in educational settings, such as schools $(n=24)$ and tertiary education $(n=11)$ focusing in non-clinical samples. Sixteen studies included participants from other community settings and two studies were conducted in mental health care facilities. Among the studies that include actual helpseekers $(n=7)$, the most common reason for seeking help was suicidal ideation, self-harm, depressive symptoms, and general mental health concerns (e.g., anxiety/ 


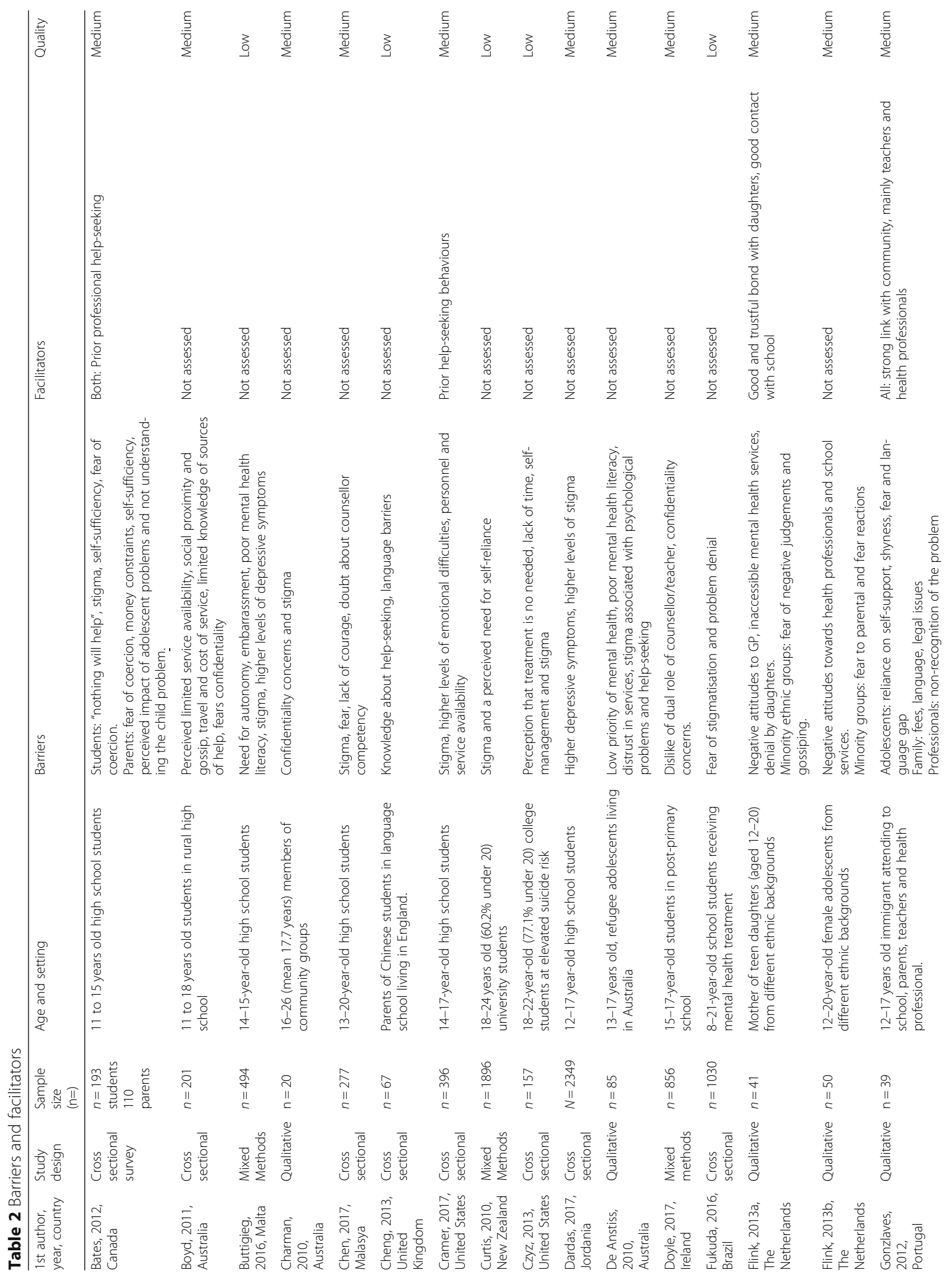




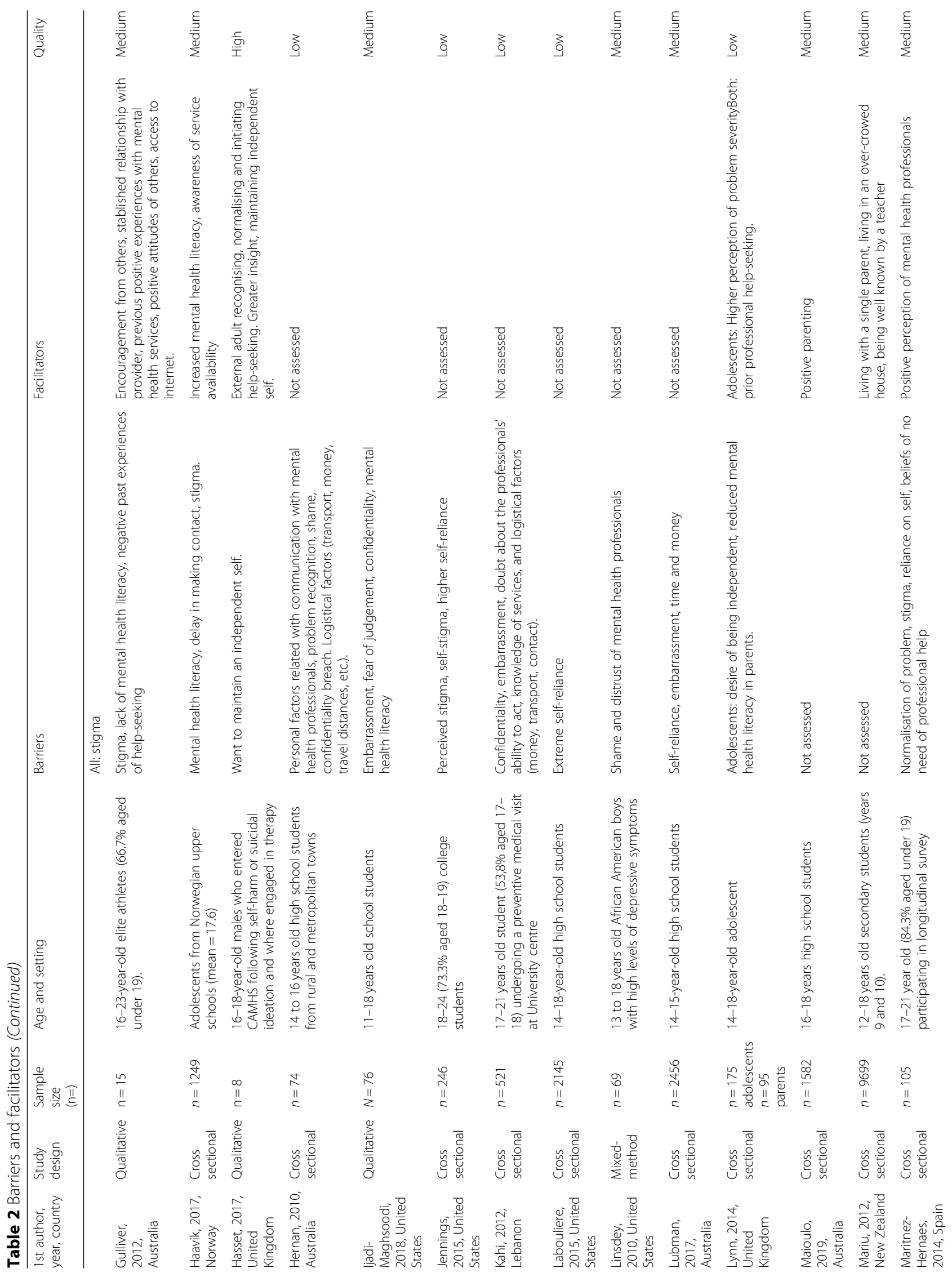




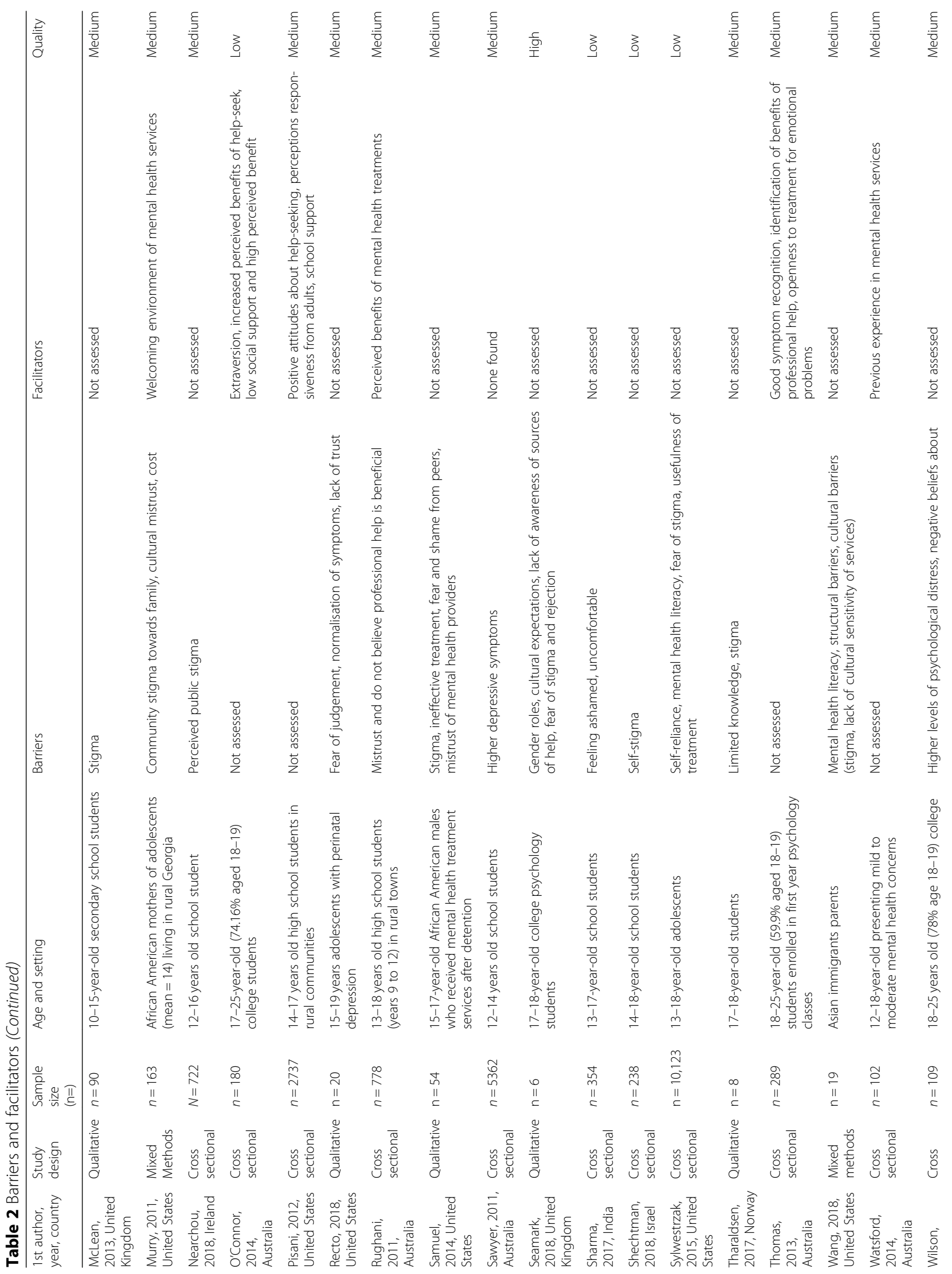




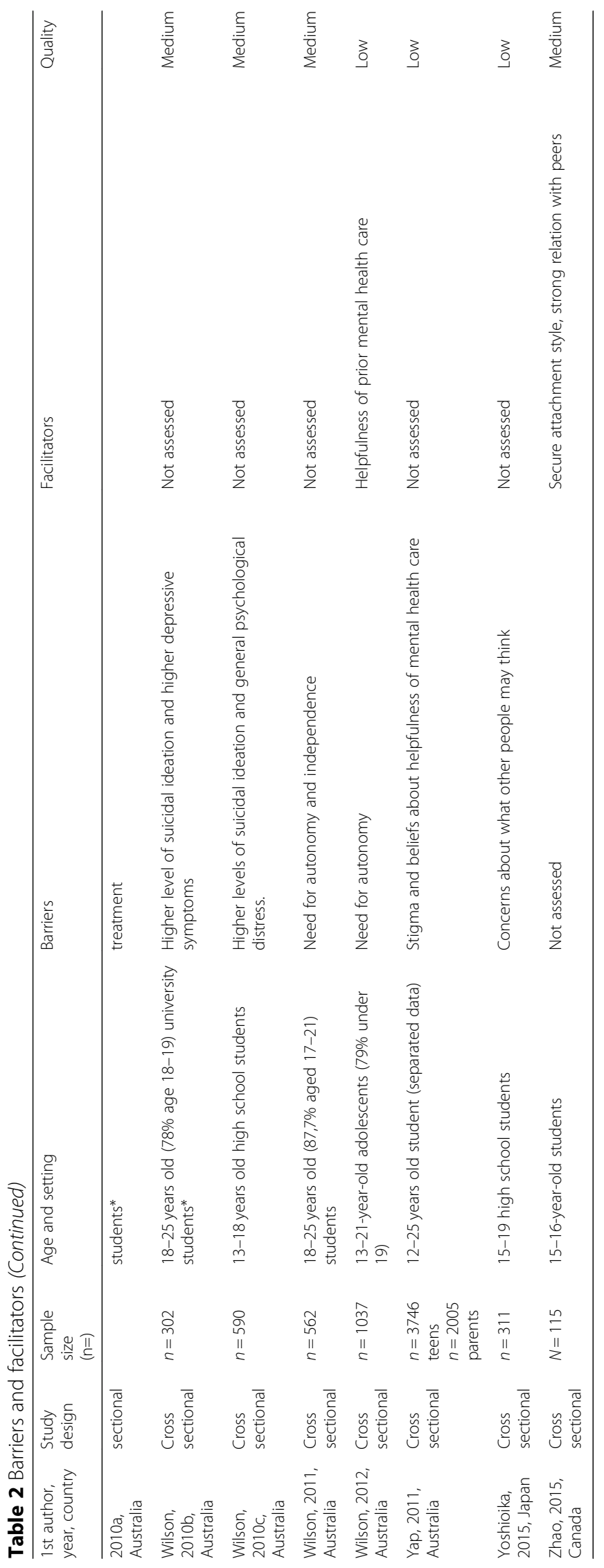




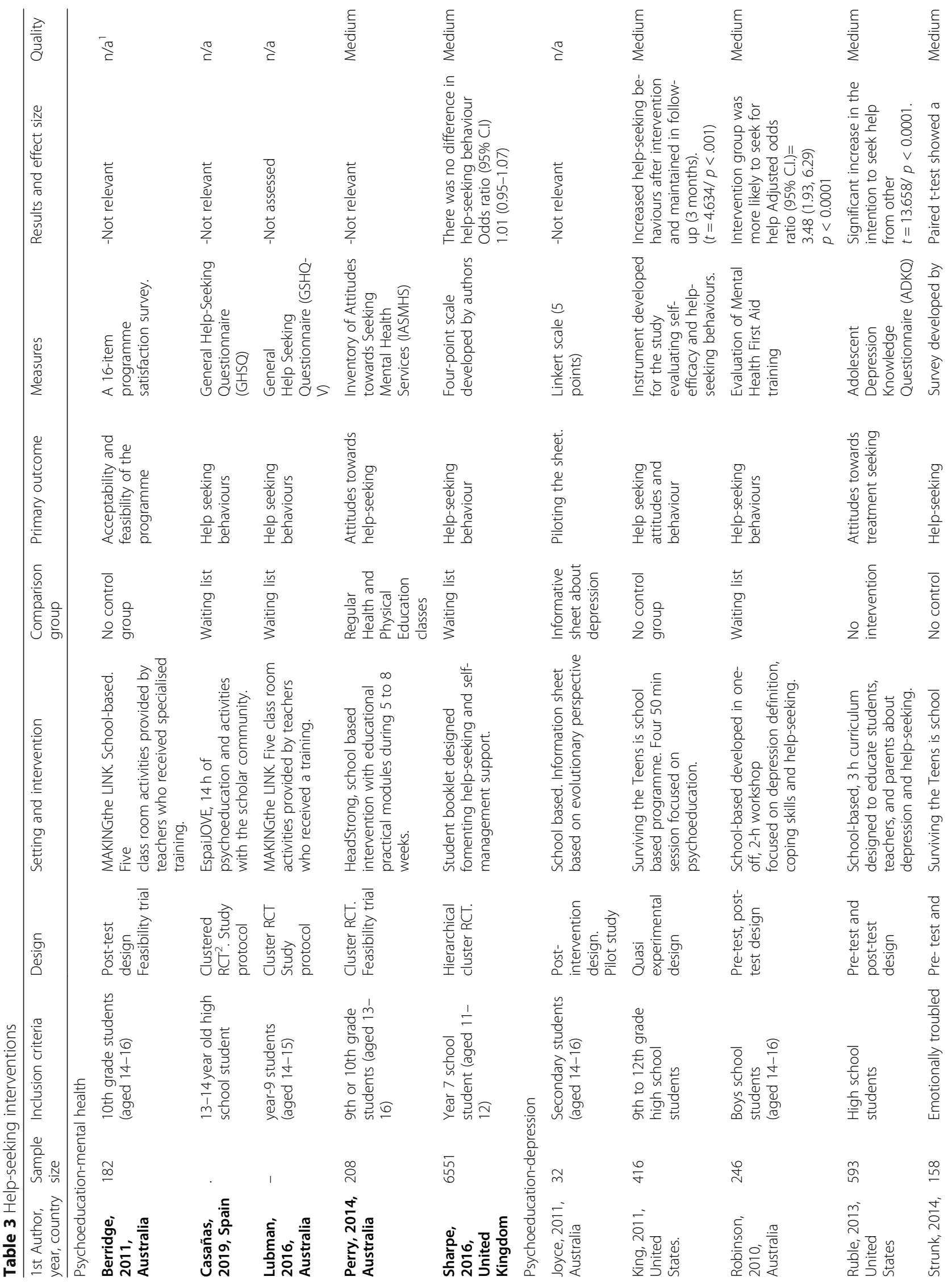




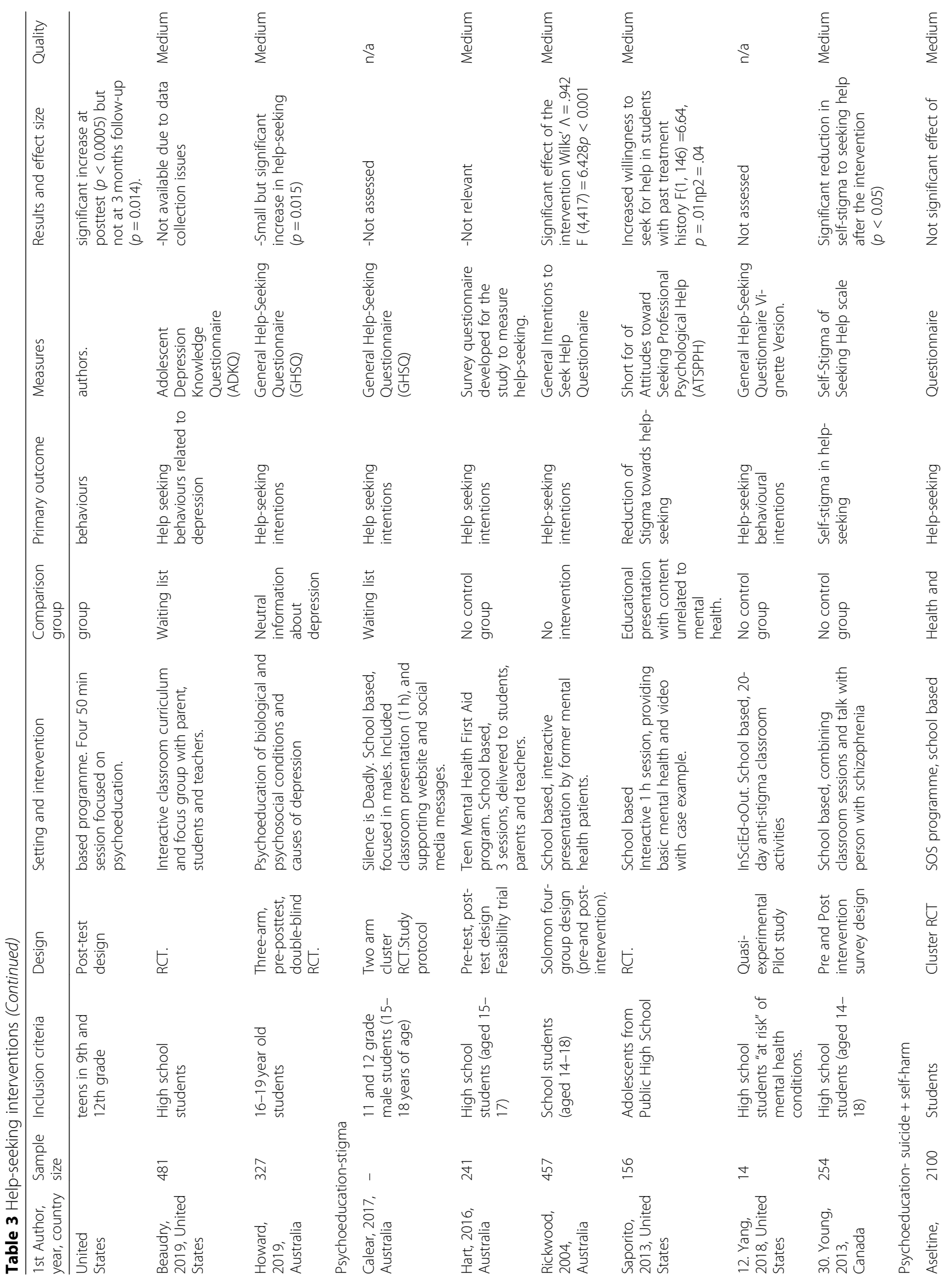




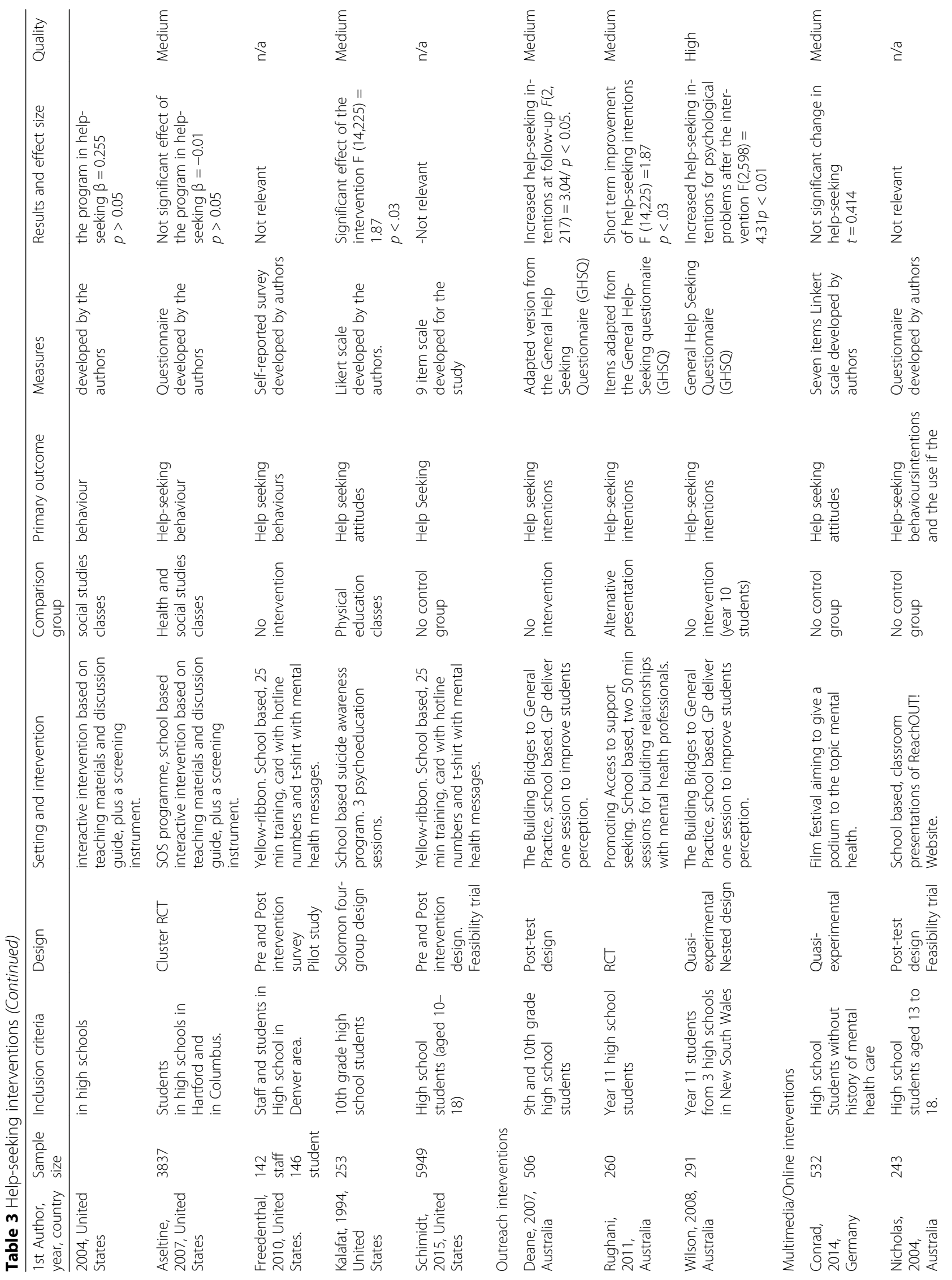




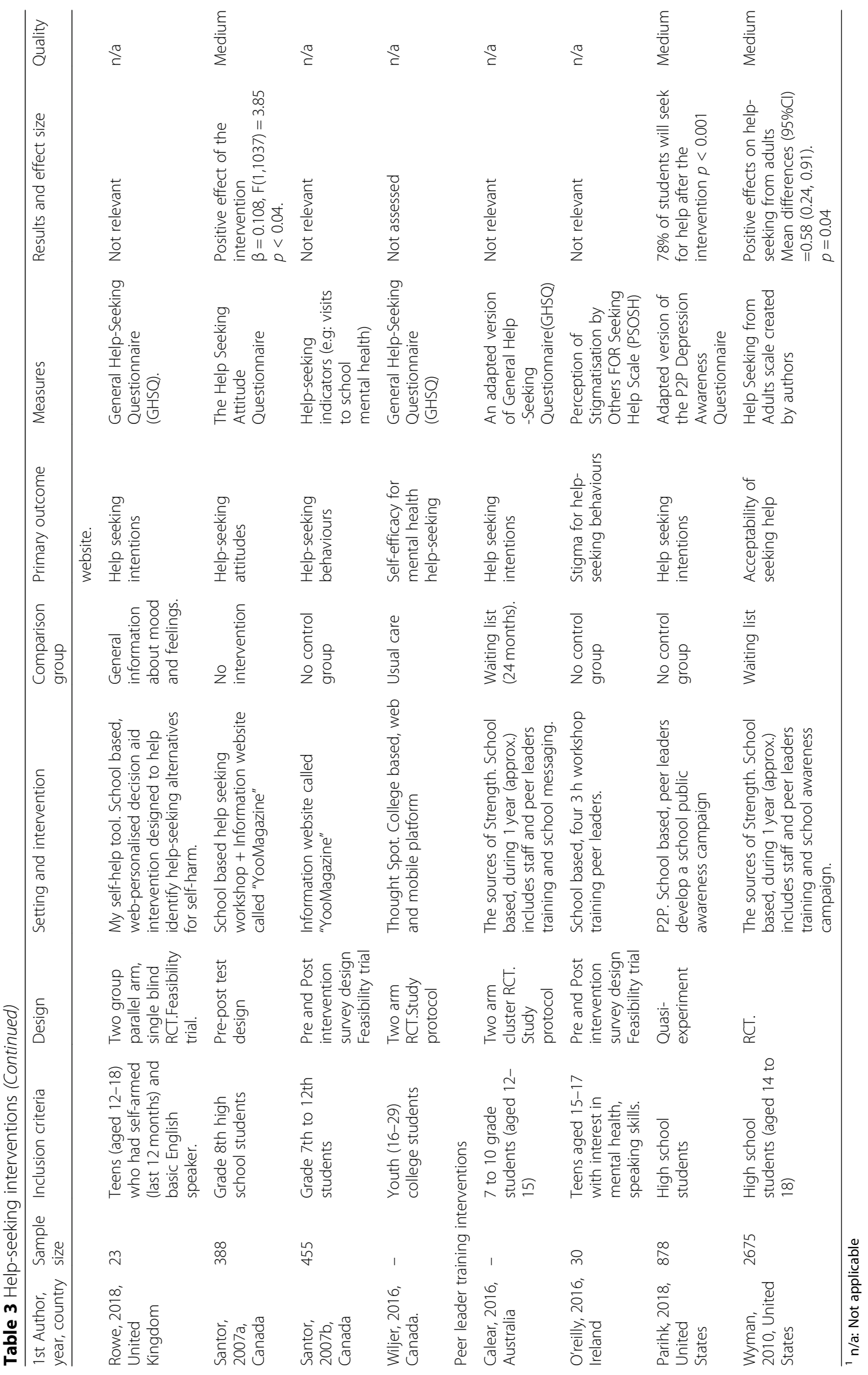


nervousness/fear). Therefore, the conclusions drawn by the majority of the articles were based on help-seeking intentions rather than actual behaviours, since the participants were not experiencing mental health problems and focused on hypothetical scenarios.

\section{Help-seeking barriers Stigma}

Stigma is defined as the fear of being socially sanctioned or disgraced leading to hiding or preventing certain actions or behaviours, including the misreporting of mental health problems [81]. More than half of the included studies $(n=30)$ made reference to this and other negative attitudes towards mental health problems as the main obstacle to help-seeking behaviours in adolescents. Of these, twenty-five studies referred to stigma as the primary obstacle, describing it through different concepts such as, "stigma", "fear of stigmatisation", "community stigma", "perceived stigma" and "self-stigma". Other negative attitudes towards mental health problems included shame, fear, and embarrassment.

\section{Family beliefs}

The second most mentioned barrier was associated to adolescents' family beliefs toward mental health services and treatment $(n=15)$. Barriers related to problem with communication and distrust towards health professionals, negative past experiences with mental health services, and believing that the treatment is not going to be helpful. This was especially true for studies including immigrant and refugee populations, which referred to cultural barriers including mistrust of mental health diagnosis and practitioners, and lack of cultural sensitivity in services as a significant barrier.

\section{Mental health literacy}

Mental health literacy refers to the ability to use mental health information to recognise, manage and prevent mental health disorders and make informed decisions about help-seeking and professional support [82]. Almost one-third of the articles $(n=14)$ referred to problems related to mental health literacy as a significant barrier including poor recognition of mental health conditions (self and others) and lack of awareness of available sources of help.

\section{Autonomy}

Adolescents' attitudes towards help-seeking revealed a perceived need of self-sufficiency and autonomy which were recognised as a relevant barrier in twelve studies, as well as fears of confidentiality breaches.

\section{Other help-seeking barriers}

To a lesser extent, problems regarding service and personnel availability and other structural factors (such as cost, transportation and waiting times) were mentioned as obstacles to help-seeking $(n=8)$. This was a significant barrier for studies including rural and immigrant populations, and in studies that included parents in their sample. Six studies focused on the relationship between symptomatology and help-seeking. These found that higher levels of psychological distress, suicidal ideation and depressive symptoms were linked to lower help-seeking behaviours.

\section{Help-seeking facilitators}

Of the 56 included studies, 19 also referred to facilitators of help-seeking behaviours. Mental health literacy and prior mental health care were the most cited facilitators for help-seeking for mental health problems $(n=10)$. Specifically, timely access to mental health was facilitated by having a previous positive experience with mental health services or help-seeking, being familiar with the sources of help, and good symptom and problem recognition. Higher engagement with the community and having a trusting and committed relationship with relevant adults such as parents, schoolteachers and counsellors also facilitated seeking help among adolescents. Further details of the included articles are available in Table 2.

\section{Secondary outcomes}

Few studies identified a significant difference when comparing younger and older adolescents in relation to barriers and facilitators to help-seeking, with no conclusive findings being reached. Some findings suggested that older adolescents tended to establish to feel more comfortable with people with mental health issues [83] and had less help-seeking fears [40]. In contrast, younger adolescents had greater knowledge about professional sources of help [34]. Only one study found a significant difference between ages regarding help-seeking, with younger adolescents reporting higher intentions of seeking help [60].

Twenty-four studies examined possible gender differences in help-seeking barriers and facilitators. Seven studies did not find significant differences between genders [28, 39, 40, 42, 46, 51, 69]. One study reported higher help-seeking intentions in males experiencing suicidal intentions [60] and two studies found that females perceived more overall barriers [26-58]. However, this may be related to higher rates of females seeking help for mental health problems compared to males [31, 33, $37,42,48,53,58,61,76]$. Studies reviewed did not evidence convincing differences between gender in relation to help-seeking. 


\section{Question 2: help-seeking interventions}

Thirty-six studies on interventions targeting helpseeking behaviour, including a total of 28,608 participants, were summarised in the review (Table 3). Most of intervention studies were conducted in Australia (14) and the United States (14), followed by Canada (4) and United Kingdom (3). All studies were conducted in educational setting including high school $(n=35)$ and college $(n=1)$. The majority of studies developed interventions for non-clinical samples, and their focus was the prevention of mental health problems and the promotion of healthy coping strategies via help-seeking behaviours. Outcomes varied between help-seeking intentions, attitudes and behaviours. Almost half of the studies focused on the effectiveness of the interventions, while sixteen were feasibility or pilot trials and study protocols. Most of the studies used a quasi-experimental design $(n=21)$ followed by randomised controlled trials $(n=15)$. The age of participants ranged from 11 to 19 years old, although one study that included participants under 29 years old was incorporated as more than half of the sample were adolescents. Interventions were delivered using four main methods: psychoeducation, outreach interventions, multimedia tools and peer leader training.

\section{Types of intervention \\ Psychoeducation}

Most of studies $(n=23)$ used psychoeducation and classroom-based interventions. Although all the interventions focused on encouraging help-seeking behaviours, the emphasis and content differed among them, including general mental health topics, suicide and depression awareness and stigma.

Five studies developed programmes based on the notion that promoting mental health awareness could enhance mental health literacy and promote helpseeking [84-88]. Four interventions targeting helpseeking for suicide were identified within five studies [89-93]. Five interventions explicitly targeted helpseeking for depression in school-based settings their focus being to educate the school population about adolescent depression and thereby encourage helpseeking [94-98]. Two studies evaluated the effectiveness of an intervention combining depression awareness and a suicide prevention programme promoting early identification and self-referral [99, 100]. Six classroom-based interventions addressing stigma were identified, two of which used psychoeducation to overcome myths regarding mental illness [101, 102] and four focused on providing interpersonal contact with people with mental health conditions in order to improve acceptance and increase helpseeking intentions [103-106].

\section{Outreach interventions}

Three studies used outreach interventions to target mental health help-seeking [107-109]. These aim to establish contact with adolescents who may be experiencing psychological and emotional distress in order to help them get the attention they need and increase their access to health services. They were based on the Building the Bridges to General Practice (BBGP) programme, developed by Wilson et al. (2005), a programme that aims to target help-seeking obstacles for physical and psychological problems by promoting contact between high school students and general practitioners [110].

\section{Multimedia interventions}

Six types of multimedia interventions have been developed to address some of the difficulties of reaching an adolescent population, such as fear of confidentiality breaches, stigma and self-reliance [111-115]. The interventions included interactive films to engage students with mental health related topic and online platforms providing personalised information regarding the decision-aids process.

\section{Peer training interventions}

Peer training interventions are focused on the training of peers who act as active agents of change and social interactions incorporated into the daily activities within the school environment [116]. All three programmes followed similar principles concerning improving the climate around mental health problems, promoting social connectedness, and challenging norms and behaviours associated with help-seeking [117-120]. "Peer leaders" acted as a link between the student population and mental health literacy, promoting the acceptability of seeking for help for mental health problems.

Further details of the included articles are available in Table 3.

\section{Secondary outcomes}

No studies referred to significant differences concerning the effectiveness of help-seeking interventions when comparing ages. No significant gender differences were identified regarding the effectiveness of the help-seeking interventions $[89,101,103,111]$. However, before the intervention, females tended to have higher mental health literacy and more adaptive attitudes regarding mental health problems [90, 111], including greater help-seeking knowledge and intentions [107, 112, 113].

\section{Effectiveness}

The main goal of this review was to describe the interventions targeting help-seeking in adolescents and therefore did not include an analysis of their effectiveness. Almost half of the included studies were study protocols 
and feasibility studies, so effect sizes were not reported. However, some findings are worth mentioning.

Four studies which looked at effectiveness of the interventions focused on psychoeducation about depression found a significant effect in increasing helpseeking. King et al., [99] identified that there was an increase in future help-seeking behaviours after the interventions and that this was maintained at 3 months' follow-up $(t=4.634 / p<.001)$. Strunk et al., [100] found a significant increase of help-seeking $(p<0.0005)$; however, this was not sustained at follow-up $(p=0.014)$. Robinson et al., [95] found that the intervention group was more likely to seek help at post-test (Odds ratio $(95 \%$ C.I $)=3.48(1.93,6.29)$, $p<0.0001)$ and Ruble et al., [96] found increased intention of help-seeking from others after the intervention $(t=13.658 / p<0.0001$. $)$.

The three studies that looked at the effectiveness of stigma reduction identified positive effects of the intervention on help-seeking. Two studies [101, 104] found a significant reduction in self-stigma surrounding seeking help after the intervention $(p<0.05)$ and one study [103] found a significant effect of the intervention in help-seeking intentions (Wilks' $\Lambda=.942, F(4,417)=$ 6.428, $p<0.001$ ).

Finally, all the studies that focused on outreach found a significant effect of the intervention in help-seeking intentions. One detected an increase in intentions at 3 months follow-up $(F(2,217)=3.04 / p<0.05)$ [108], Rughani [107] found short terms improvements in helpseeking intentions $(\mathrm{F}(14,225)=1.87 p<.03)$ and Wilson [109] found a significant effect in the intention of seeking help for psychological problems after the intervention $(\mathrm{F}(2,598)=4.31 p<0.01)$.

\section{Quality assessment}

The majority of the studies were low to medium quality with moderate to high risk of bias. Most of the crosssectional studies did not state a clear inclusion and exclusion criteria and did not consider possible confounders affecting the interpretation of the outcome. Regarding qualitative research, the most common problem was linked to sample size and the difficulty of providing a clear strategy to address the subjectivity of the authors in the interpretations of the data. Mixed method studies presented some inconsistencies in addressing specific components of both quantitative and qualitative traditions, and in the process of integrating both approaches. Regarding intervention studies, it was difficult to identify to what extent the groups were similar at baseline. Although some studies included baseline measures of demographic information, most of them did not consider confounders or other factors influencing effectiveness, and some studies did not have any baseline measures. Also, few studies included follow-up and the ones that did, had high attrition rates and short followup periods (up to 6 months); therefore, it is not possible to attribute a long-lasting effect to the interventions. Quasi-experimental studies acknowledge possible selection and sample bias. Randomised controlled trials presented difficulties in terms of the blinding of the research team and participants at different stages of the process.

Overall there was inconsistency regarding the measurements of help-seeking, with most of the studies focusing on help-seeking intentions, which is not necessarily related to future behaviours. Moreover, many studies did not use valid and reliable instruments for measuring help-seeking. This is especially true for the experimental studies since most of them developed tools focused on their intervention rather than standardised help-seeking measures. Finally, most of the studies only used self-report measures, increasing the risk of bias of the findings. We did not assess the quality of study protocol, feasibility studies and pilot studies.

\section{Discussion}

\section{Question 1: barriers and facilitators}

This review focused on identifying barriers, facilitators and interventions targeting help-seeking behaviours in adolescents. Consistent with previous findings [1], the most prominent barrier identified was stigma. Negative attitudes and beliefs about mental health services and professionals was the second most prominent barrier. Trusted and strong relationships with possible gatekeepers (teachers, parents, GPs, health professionals, etc.) and prior positive help-seeking experience were the most cited facilitators.

Few studies related symptom severity with helpseeking. Of those that did, higher symptomatology was associated with lower help-seeking intentions and behaviours. This is in line with previous studies suggesting that teens who are most in need are less likely to seek help $[1,11,15]$. It is possible that the nature of mental health symptoms such as self-blame, emotional distress, difficulty in speaking to others and diminished cognitive ability contribute to lower help-seeking behaviours. Adolescents with higher symptom severity may be even more vulnerable experiencing difficulties with the helpseeking process in areas such as identifying the need for professional assistance or fear of stigmatisation. This could be due to higher rates of isolation and exclusion from their peers. Increasing mental health literacy among this population may provide a way of improving social support between peers [121].

There are structural barriers affecting the help-seeking process that go beyond attitudes, for example, costs, waiting times and transportation. These barriers were 
not among the most prominent reasons cited in the research review; however, this may be related to the limited amount of studies that included parents' perceptions. A previous review, which focused on the parents of children and adolescents, concluded that structural barriers were the most relevant [122]. This suggests that adolescents are less worried about the practical implications of accessing help for mental health problems and are more affected by being attitudinal barriers, but that structural barriers may be more relevant to parents.

Key facilitators to help-seeking should be considered when creating new interventions such as trusted relationships with gatekeepers, and familiarisation with the help-seeking process. However, the lack of studies focusing on facilitators precludes many conclusions being drawn. The majority of studies used sub-clinical samples and/or hypothetical help-seeking scenarios rather than asking genuine help-seeker with mental health problems who could refer to the real circumstances leading them to ask for help. More research including young people who have sought help from services would be useful in understanding the idiosyncrasies of this process.

These findings provide a useful overall picture of the relevant factors influencing the help-seeking process in adolescents. However, the included studies did not share a clear definition and framework regarding help-seeking. A wide range of tools were used to measure helpseeking, varying in their validity and reliability, and also in the constructs they measured. This limits the generalisability of the findings and our understanding of the help-seeking process. Rickwood \& Thomas (2012) have proposed a framework regarding help-seeking, identifying the different parts of the process, sources of help, types of help and main concerns [15]. In the future, sharing such a framework could be a useful means to reach a general agreement regarding the definition of help-seeking and its components.

\section{Question 2: interventions}

The types of interventions varied considerably and included classroom-based psychoeducation, outreach interventions, multimedia and online-based interventions and peer training. Among classroom-based psychoeducation interventions, the most effective ones were those focused on prompting help-seeking through addressing depression and stigma. All peer outreach interventions had a significant effect in improving help-seeking intentions, thus showing promising results. In sum, addressing stigma, mental health literacy, and attitudes towards mental health services could be beneficial in terms of promoting help-seeking.
Most of the intervention studies included in this review did not investigate mechanisms of change with regards to help-seeking behaviour. The relevance of studying underlying mechanisms and practical requirements related to the functionality of interventions has been previously discussed [123], and most of the interventions included in this review did not refer to these processes. Identifying such mechanisms could help understand how interventions work, enlightening and optimising the process of decision-making and design [93]. Adolescence is a period essentially characterised by emotional, behavioural, hormonal, and neuronal changes $[124,125]$. Interventions congruent with the developmental stages may be useful to target age-appropriate factors.

It is important to mention that few intervention studies referred in detail to the implementation process and the main issues they encountered; however, the studies which did refer to this, found significant barriers. School administration issues, the difficulty of obtaining parental consent and attrition rates for the follow-up measures were one of the main difficulties regarding the implementation [93, 97, 102, 115]. Teacher's support and engagement with the intervention were also described as a barrier in the implementation process for some studies $[85,93,114]$. Most of studies concluded that implementation strategies should consider the reality and challenges of each school. For this the theme of contextualization is fundamental and the specificities of the process of implementation (planning, engaging, executing, reflecting and evaluating) $[126,127]$.

All interventions were conducted within an educational setting. Special attention should also be paid to young people outside of the educational system, who are particularly vulnerable in terms of economic and social deprivation [128]. Around one in five children and adolescents are out of school according to the UNESCO [129], with psychosocial factors appearing to obstruct traditional educational trajectories [130]. Health and mental health conditions have a relevant role in terms of absenteeism and truancy [131]. Adolescents experiencing symptoms of depression and anxiety or in charge of a chronically sick relative can be more prone to avoid school and stay at home. These children can be even more vulnerable and harder to reach, and there is a lack of collaborative effort attempting to overcome this situation. Encouraging partnerships between the health and educational systems, community settings, youth detention centres, among other institutions providing social care, should be promoted with the purpose of supporting mental healthcare and provision for young people [132].

Encouraging adolescents to seek help for mental health problems is a key priority however, this does not 
resolve the discrepancy between needs and resources worldwide [132, 133]. "Mental health services for children and adolescents have internationally been poorly understood, underfunded and even neglected by governments" [p.92, 134]. This may be associated with the lack of a general understanding of this population's needs (including developmental issues), and the "implementation gap", referring to the challenges of translating evidence to health service development and practice [134]. Simultaneously, focusing on increasing help-seeking and service availability for children and adolescents is necessary to reduce the global burden of disease and protect the future health of this population $[125,135]$.

\section{Limitations}

This review has a number of limitations. First, only one author performed the data extraction and critical appraisal of papers therefore the data analysis is at risk of some subjectivity. Second, there is an increasing debate regarding the age that adolescence comprises, with some suggesting the age should be extended to 10 to 24 years old [17]. However, we decided to follow the definition of 'adolescent' established by international organisations including the OMS and UNICEF. A significant number of papers were excluded considering our age range $(n=104)$. Defining adolescence as a period between 10 to 19 years old could be a limitation to our study. Thirdly, this review focused on common mental health problems such as depression, anxiety and emotional distress and excluded psychiatric conditions such as anorexia, schizophrenia and substance misuse, mainly due to the particular nature of the help-seeking processes. However, the exclusion of substance misuse problems could be seen as a limitation of this study due to its high prevalence in adolescence, making it a particularly sensitive issue during this period of life [136]. Finally, this review prioritised the overinclusion of studies to have an overall picture of the existing evidence regarding helpseeking for mental health problems in adolescents. As a result, low quality studies were included in the analysis and may affect the interpretation of the findings. There were some notable strengths in this review. This is the first systematic review studying help-seeking barriers, facilitators and interventions in order to give a comprehensive review of the topic. The search strategy developed was over-inclusive, using an optimal database combination, including multiple languages and PPI involvement in the development of the topic.

\section{Conclusion}

In conclusion, stigma and negative beliefs about mental health services appear as the most significant barriers to help-seeking for adolescents, whereas previous positive experiences with services and good mental health literacy are the most relevant facilitators. There are a number of interventions being developed to promote help-seeking for mental health problems in adolescents, and most of them take place in high education settings. They include a range of delivery methods including psychoeducation, stigma and depression awareness campaigns, online tools and peer training. Since such initiatives are relatively new, there is a need for more trials, with longer follow-up periods and the use of reliable and validated tools focused in future help-seeking behaviour. Despite school seeming to be the ideal setting for deploying these interventions, it is important to consider adolescents outside the school system who may be in more need of attention for psychosocial and mental health problems.

\section{Supplementary information}

Supplementary information accompanies this paper at https://doi.org/10. 1186/s12888-020-02659-0.

\section{Additional file 1.}

Abbreviations

MMAT: Mixed Methods Appraisal Tool; RCT: Randomised Controlled Trial

\section{Acknowledgements}

The completion of this systematic review would not have been possible without the support of The National Commission of Scientific and Technological Research (CONICYT-Beca Chile) under the Ministry of Education of the Chilean Government.

\section{Authors' contributions}

The contributions of the authors are as follows: AA developed the review protocol, was the first screener and drafted the paper; IS was the second screener for this review; JB commented on the draft of the paper; MJ was the third contributor available to discuss any discrepancies between the two screeners; SR was the senior author, and was involved the design of the review questions, the protocol and commented on the draft of the paper. The author(s) read and approved the final manuscript.

Funding

N/A. This review was conducted as a dissertation in the context of the MSc Clinical Mental Health Sciences at UCL.

Availability of data and materials

Data sharing is not applicable to this article as no datasets were generated or analysed during the current study.

Ethics approval and consent to participate

This is a systematic review including studies based on human participants. All the included papers had ethical approval, consent to participate and consent for publication authorised by the participants.

\section{Consent for publication}

See above.

\section{Competing interests}

Sarah Rowe has been involved in developing a help-seeking intervention for adolescent self-harm (https://bmjopen.bmj.com/content/6/9/e012161).

\section{Author details}

${ }^{1}$ Child and Adolescent Mental Health Service, Children's Hospital Dr. Roberto del Río, Santiago, Chile. ${ }^{2}$ Public Health School, Faculty of Medicine, University of Chile, Santiago, Chile. ${ }^{3}$ División of Psychiatry, University College London, 
London, UK. ${ }^{4}$ Camden and Islington Mental Health Foundation Trust, London, UK.

\section{Received: 23 September 2019 Accepted: 11 May 2020 Published online: 11 June 2020}

\section{References}

1. Gulliver A, Griffiths K, Christensen H. Perceived barriers and facilitators to mental health help-seeking in young people: a systematic review. BMC Psychiatry. 2010;12:81.

2. WHO: Adolescence Mental Health. Mapping actions of nongovernmental associations and other international development organisations. [Online]. Geneva: World Health Organisation; 2012. Available from: http://apps.who. int/iris/bitstream/handle/10665/44875/9789241503648_eng.pdf;jsessionid= B09D1D46A7AA36AA5757E9E191B3F023? sequence=1. Accessed 11 Sept 2019.

3. WHO. Mental health: strengthening our response. Geneva: World Health Organisation. Available from: https://www.who.int/news-room/fact-sheets/ detail/mental-health-strengthening-our-response.

4. WHO. Adolescents: health risks and solutions. Geneva: World Health Organisation; 2018. Available from: http://www.who.int/en/news-room/factsheets/detail/adolescents-health-risks-and-solutions Accessed 09 May 2018

5. Murphy, M. \& Fonagy, P. Our children deserve better, prevention pays: annual report of the Chief Medical Officer. London: Department of Health. 2013. Chapter 10: Mental health problems in children and young people

6. Michaud P, Fombonne $\mathrm{E}$. The $\mathrm{ABC}$ of adolescence: common mental health problems. BMJ. 2005;330(7495):835-8. https://doi.org/10.1136/bmj. 330.7495 .835

7. Oksanen A, Laimi K, Bjorklund K, Löyttyniemi E, Kunttu K. A 12-year trend of psychological distress: national study of Finnish University students. Cent Eur J Public Health. 2017;25(2):113-9. https://doi.org/10.21101/cejph.a4438.

8. Kowadenko N, Culjak G. Workforce planning for children and young people's mental health care. Lancet Public Health. 2018;3:266-7.

9. Children Comissioners. Lighting review: access to child and adolescent mental health services. United Kingdom: Children Comissioners; 2016. Available from:https://www.childrenscommissioner.gov.uk/wp-content/ uploads/2017/06/Childrens-Commissioners-Mental-Health-LightningReview.pdf.

10. Rowe S, French R, Henderson C, Ougrin D, Slade M, Moran P. Help-seeking behaviour and adolescent self-harm: a systematic review. Aust N Z J Psychiatry. 2014;48(12):1083-95.

11. Divin N, Harper P, Curran E, Corry D, Leavey G. Help-seeking measures and their use in adolescents: a systematic review. Adolesc Res Rev. 2018;3(1): 113-22. https://doi.org/10.1007/s40894-017-0078-8.

12. Rickwood D, Thomas K. Conceptual measurement framework for helpseeking for mental health problems. Psychol Res Behav Manag. 2012;5:17383.

13. Rickwood D, Deane F, Wilson C. When and how do young people seek professional help for mental health problems? Med J Aust. 2007;187(7):35-9.

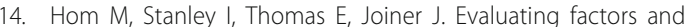
interventions that influence help-seeking and mental health service utilization among suicidal individuals: a review of the literature. Clin Psychol Rev. 2015:40:28-39.

15. Rickwood D, Deane F, Wilson C, Ciarrochi J. Young people's help-seeking for mental health problems. Aust J Adv Ment Health. 2005:4(3):218-51.

16. Gulliver A, Griffiths KM, Christensen H, Brewer JL. A systematic review of help-seeking interventions for depression, anxiety and general psychological distress. BMC Psychiatry. 2012;12:12-81.

17. Xu Z, Huang F, Kosters M, Staiger T, Becker T, Thornicroft G, Rusch N. Effectiveness of interventions to promote help-seeking for mental health problems: systematic review and meta-analysis. Psychol Med. 2018;48(16): 2658-67.

18. Sawyer S, Azzopardi P, Wickremarathne D, Patton G. Viewoint: the age of adolescence. Lancet. 2018;2(3):223-8. https://doi.org/10.1016/S23524642(18)30022-1.

19. The PRISMA Group. PRISMA statement. Oxford: University of Oxford; 2009. Available from:http://prisma-statement.org/prismastatement/FlowDiagram. aspx. Accessed 30 May 2018

20. Bramer W, Rethlefsen M, Leijnen J, Franco O. Optimal database combination for literature search and systematic review: a prospective exploratory study. Syst Rev. 2017;6:245. https://doi.org/10.1186/s13643-017-0644-y.
21. Joana Brigss Institute. Critical assessment tools. Melbourne: The Joana Briggs Institute. Available at: http://joannabriggs.org/critical_appraisal tools.

22. Hong Q, Pluye P, Fabruegues S, Bartlett G, Boardman F, Cargo M, Dagenais P, Gagnon MP, Griffiths F, Nicolau B, O'Cathain A, Rousseau MC, Vedel I. Mixed methods appraisal tool (MMAT). Montreal: McGill University Department of Family Medicine; 2018. User quide.

23. Munn Z, Moola S, Ritjano D, Lisy K. The development of a critical appraisal tool for use in systematic reviews addressing questions of prevalence. Int J Health Policy Manag. 2014;3:123-8.

24. Pluye P. Critical appraisal tools for assessing the methodological quality of qualitative, quantitative and mixed methods studies included in systematic mixed studies reviews. EBM. 2012;19(4):29-46. https://doi.org/10.1111/jep.12017.

25. Popay, J. Roberts, H. Sowden, A. Petticrew, M. Arai, L. Rodgers, M. Britten, N. Roen, K. Duffy, R. Guidance on the conduct of narrative synthesis in systematic reviews. National Centre for Research Methods, United Kingdom. [Online] Available from: http://citeseerx.ist.psu.edu/viewdoc/download?doi= 10.1.1.178.3100\&rep=rep1\&type $=$ pdf.

26. Hernan A, Philpot B, Edmonds A, Reddy P. Healthy minds for country youth: help-seeking for depression among rural adolescents. Aust J Rural Health 2010;18(3):118-24. https://doi.org/10.1111/j.1440-1584.2010.01136.x.

27. Wilson CJ. General psychological distress symptoms and help-avoidance in young Australians. Adv Ment Health. 2010;9(1):63-72.

28. Wilson CJ, Deane F. Help-negation and suicidal ideation: the role of depression, anxiety and hopelessness. J Youth Adolesc. 2010;39(3):291-305. https://doi.org/10.1007/s10964-009-9487-8.

29. Wilson CJ, Deane FP, Marshall KL, Dalley A. Adolescents' suicidal thinking and reluctance to consult general medical practitioners. J Youth Adolesc. 2010;39(4):343-56. https://doi.org/10.1007/s10964-009-9436-6.

30. Boyd CP, Hayes L, Nurse S, Aisbett D, Francis K, Newnham K, Sewell J. Preferences and intention of rural adolescents toward seeking help for mental health problems. Rural Remote Health. 2011;11(1):1582.

31. Rughani J, Deane FP, Wilson CJ. Rural adolescents' help-seeking intentions for emotional problems: the influence of perceived benefits and stoicism. Aust J Rural Health. 2011;19(2):64-9. https://doi.org/10.1111/j.1440-1584. 2011.01185.x.

32. Wilson CJ, Rickwood DJ, Bushnell JA Caputi P. Thomas SJ. The effects of need for autonomy and preference for seeking help from informal sources on emerging adults' intentions to access mental health services for common mental disorders and suicidal thoughts. Adv Ment Health. 2011;10(1):29-38.

33. Sawyer MG, Borojevic N, Ettridge KA, Spence SH, Sheffield J, Lynch J. Do help-seeking intentions during early adolescence vary for adolescents experiencing different levels of depressive symptoms? J Adolesc Health. 2011:50(3):236-42

34. Yap MB, Wright A, Jorm AF. The influence of stigma on young people's help-seeking intentions and beliefs about the helpfulness of various sources of help. Soc Psychiatry Psychiatr Epidemiol. 2011;46(12):1257-65.

35. Bates $\sqcup$. Predictors of help seeking among early adolescents: stages and factors. Dissertation Abstracts International: Section B: Sci Eng. 2012;73(4-B):2539.

36. Cheng WSW, Fenn D, Couteur AL. Understanding the mental health needs of Chinese children living in the north east of England. Ethn Inequalities Health Soc Care. 2013:6(1):16-22.

37. Kahi HA, Abi Rizk GY, Hlais SA, Abid SM. Health-care-seeking behaviour among university students in Lebanon. East Mediterr Health J. 2012;18(6): 598-606.

38. Mariu KR, Merry SN, Robinson EM, Watson PD. Seeking professional help for mental health problems, among New Zealand secondary school students. Clin Child Psychol Psychiatry. 2012;17(2):284-97. https://doi.org/10.1177/ 1359104511404176

39. Pisani AR, Schmeelk-Cone K, Gunzler D, Petrova M, Goldston DB, Tu X Wyman PA. Associations between suicidal high school Students' helpseeking and their attitudes and perceptions of social environment. J Youth Adolesc. 2012:41(10):1312-24. https://doi.org/10.1007/s10964-012-9766-7.

40. Wilson CJ, Deane FP. Brief report: need for autonomy and other perceived barriers relating to adolescents' intentions to seek professional mental health care. J Adolesc. 2012;35(1):233-7

41. Czyz EK, Horwitz AG, Eisenberg D, Kramer A, King CA. Self-reported barriers to professional help seeking among college students at elevated risk for suicide. J Am Coll Heal. 2013:61(7):398-406.

42. Thomas SJ, Caputi P, Wilson CJ. Specific attitudes which predict psychology Students' intentions to seek help for psychological distress. J Clin Psychol. 2013;70(3):273-82 
43. Lynn J. Factors predicting adolescents and parents help seeking behaviour. [Online] Windsor: University of Windsor. 2014. (Doctoral dissertation). Available from: https://pdfs.semanticscholar.org/27aa/3be1f04f223a2c2d84 ee8ecdbff24baaf942.pdf.

44. Martinez-Hernaez A, DiGiacomo SM, Carceller-Maicas N, Correa-Urquiza M, Martorell-Poveda MA. Non-professional-help-seeking among young people with depression: a qualitative study. BMC Psychiatry. 2014;14(124). https:// doi.org/10.1186/1471-244x-14-124.

45. O'Connor P, Martin B, Weeks CS, Ong L. Factors that influence young people's mental health help-seeking behaviour: a study based on the health belief model. J Adv Nurs. 2014;70(11):2577-87.

46. Watsford C, Rickwood D. Young people's expectations, preferences, and experiences of therapy: effects on clinical outcome, service use, and helpseeking intentions. Clin Psychol. 2014;18(1):43-51. https://doi.org/10.1111/ cp.12034.

47. Jennings KS, Cheung JH, Britt TW, Goguen KN, Jeffirs SM, Peasley AL, Lee AC. How are perceived stigma, self-stigma, and self-reliance related to treatment-seeking? A three-path model. Psychiatr Rehabil J. 2015;38(2):10916.

48. Labouliere CD, Kleinman M, Gould MS. When self-reliance is not safe: associations between reduced help-seeking and subsequent mental health symptoms in suicidal adolescents. Int J Environ Res Public Health. 2015; 12(4):3741-55.

49. Sylwestrzak A, Overholt CE, Ristau Kl, Coker KL. Self-reported barriers to treatment engagement: adolescent perspectives from the National Comorbidity Survey-Adolescent Supplement (NCS-A). Community Ment Health J. 2015;51(7):775-81. https://doi.org/10.1007/s10597-0149776-x.

50. Yoshioka K, Reavley NJ, Hart LM, Jorm AF. Recognition of mental disorders and beliefs about treatment: results from a mental health literacy survey of Japanese high school students. Int J Cult Ment Health. 2015;8(2):207-22.

51. Zhao WF, Young RE, Breslow L, Michel NM, Flett GL, Goldberg JO. Attachment style, relationship factors, and mental health stigma among adolescents. Can J Behav Sci. 2015:47(4):263-71. https://doi.org/10.1037/ cbs0000018.

52. Fukuda CC, Penso MA, do Amparo DM, de Almeida BC, de Aquino Morais C. Mental health of young Brazilians: barriers to professional help-seeking. Estud Psicol. 2016;33(2):355-65.

53. Lubman D, Cheetham I, Jorm A, Berridge AF, Wilson BJ, Blee C, Proimos F, J. Australian adolescents' beliefs and help-seeking intentions towards peers experiencing symptoms of depression and alcohol misuse. BMC Public Health. 2017;17:658. https://doi.org/10.1186/s12889-017-4655-3.

54. Sharma M, Banerjee B, Garg S. Assessment of mental health literacy in school-going adolescents. J Indian Assoc Child Adolesc Ment Health. 2017; 13(4):263-83.

55. Cramer, K. Mental health help seeking in schools: the impact of mental health literacy, stigma, and barriers to care. Dissertation abstracts international section A: humanities and social sciences; 2017;78(3-A(E)), No Pagination Specified.

56. Chen KS, Kok JK. Barriers to seeking school counselling: Malaysian Chinese school students' perspectives. J Psychol Couns Sch. 2017;27(2):222-38. https://doi.org/10.1017/jgc.2015.21.

57. Dardas, L. Silva, S. Van De Water, B. Vance, A. Smoski, M. Noonan, D. Simmons, L. (2017). Psychosocial correlates of Jordanian adolescents' helpseeking intentions for depression: findings from a nationally representative school survey. J Sch Nurs. DOI: https://doi.org/10.1177/1059840517731493.

58. Haavik L, Joa I, Hatloy K, Stain HJ, Langeveld J. Help seeking for mental health problems in an adolescent population: the effect of gender. J Ment Health. 2017;28(5):467-74.

59. Shechtman Z, Vogel DL, Strass HA, Heath PJ. Stigma in help-seeking: the case of adolescents. Br J Guid Couns. 2018;46(1):104-19. https://doi.org/10. 1080/03069885.2016.1255717.

60. Nearchou F, Bird N, Costello A, Duggan S, Gilroy J, Long R, McHuhg L, Hennesey E. Personal and perceived public mental health stigma as predictors of help-seeking intentions in adolescents. J Adolesc. 2018. https://doi.org/10.1016/j.adolescence.2018.05.003.

61. Maiuolo M, Deane F, Ciarrochi J. Parental authoritativeness, social support and help-seeking for mental health problems in adolescents. J Youth Adolesc. 2019. https://doi.org/10.1007/s10964-019-00994-4.

62. Charman D, Harms C, Myles-Pallister J. Help and e-help - young people's perspectives of mental healthcare. Aust Fam Physician. 2010;39(9):663-5.
63. De Anstiss H, Ziaian T. Mental health help-seeking and refugee adolescents: qualitative findings from a mixed-methods investigation. Aust Psychol. 2010; 45(1):29-37

64. Flink IJ, Beirens TM, Butte D, Raat $H$. The role of maternal perceptions and ethnic background in the mental health help-seeking pathway of adolescent girls. J Immigr Minor Health. 2013;15(7):292-9.

65. Flink IJ, Beirens TM, Butte D, Raat H. Help-seeking behaviour for internalizing problems: perceptions of adolescent girls from different ethnic backgrounds. Ethn Health. 2013;19(2):160-77.

66. Gulliver A, Griffiths KM, Christensen H. Barriers and facilitators to mental health help-seeking for young elite athletes: a qualitative study. BMC Psychiatry. 2012;12(157). https://doi.org/10.1186/1471-244x-12-157.

67. Goncalves M, Moleiro C. The family-school-primary care triangle and the access to mental health care among migrant and ethnic minorities. J Immigr Minor Health. 2012;14(4):682-90. https://doi.org/10.1007/s10903-0119527-9.

68. Hassett A, Isbister C. Young men's experiences of accessing and receiving help from child and adolescent mental health services following self-harm. SAGE Open. 2017;7(4). https://doi.org/10.1177/2158244017745112

69. McLean A, Hunt K, Sweeting H. Symptoms of mental health problems: children's and adolescents' understandings and implications for gender differences in help seeking. Child Soc. 2013;13:161-73.

70. Samuel, I. A. Disparities in mental health service use among African American adolescent males released from juvenile detention facilities. Dissertation abstracts international section A: humanities and social sciences; 2014; 75(5-A(E)), No Pagination Specified.

71. Tharaldsen KB, Stallard P, Cuijpers P, Bru E, Bjaastad JF. 'It's a bit taboo': a qualitative study of Norwegian adolescents' perceptions of mental healthcare services. Emot Behav Diffic. 2017;22(2):111-26. https://doi.org/10. 1080/13632752.2016.1248692.

72. Seamark D, Gabriel L. Barriers to support: a qualitative exploration into the help-seeking and avoidance factors of young adults. Br J Guid Couns. 2018; 46(1):120-31. https://doi.org/10.1080/03069885.2016.1213372.

73. Ijadi-Maghsoodi R, Bonnet K, Feller S, Nagaran K, Puffer M, Kataoka S. Voices from minority youth on help-seeking and barriers to mental health services: partnering with school-based health centers. Ethn Dis. 2018;28(2):437-44. https://doi.org/10.18865/ed.28.S2.437.

74. Recto P, Champion J. "We don't want to be judged": perceptions about professional help and attitudes towards help-seeking among postpartum and pregnant Mexican-American adolescents. J Pedatr Nurs. 2018;42:111-7. https://doi.org/10.1016/j.pedn.2018.04.010.

75. Buttigieg A, Camilleri M, Calleja N. Barriers to mental health help-seeking behaviour in adolescents in Malta. Eur J Pub Health 2016:26:379.

76. Curtis C. Youth perceptions of suicide and help-seeking: 'They'd think I was weak or "mental"'. J Youth Stud. 2010;13(6):699-715.

77. Doyle L, Treacy MP, Sheridan A. 'It just doesn't feel right': a mixed methods study of help-seeking in Irish schools. Adv School Ment Health Promot. 2017;10(2):113-26. https://doi.org/10.1080/1754730x.2017.1285710.

78. Lindsey MA, Joe S, Nebbitt V. Family matters: the role of mental health stigma and social support on depressive symptoms and subsequent help seeking among African American boys. J Black Psychol. 2010;36(4):458-82. https://doi.org/10.1177/0095798409355796.

79. Murry VM, Heflinger CA, Suiter SV, Brody GH. Examining perceptions about mental health care and help-seeking among rural African American families of adolescents. J Youth Adolesc. 2011;40(9):1118-31. https://doi.org/10.1007/ s10964-010-9627-1.

80. Wang C, Do K, Freese K, Zheng L. Asian immigrants perceptions of barriers preventing adolescents from seeking school-based mental health services. Sch Ment Heal. 2018. https://doi.org/10.1007/s12310-018-9285-0.

81. Bharadwaj P, Pai MM, Suziedelyte A. Mental health stigma. Econ Lett. 2017; 159:57-60. https://doi.org/10.1016/j.econlet.2017.06.028.

82. Kutcher S, Wei Y, Coniglio C. Mental health literacy: past, present and future Can J Psychiatr. 2016;61(3). https://doi.org/10.1177/0706743715616609.

83. Conrad I, Schulze B, Corrieri S, Heider D, Schomerus G, Riedel-Heller SG. The film festival "AUSNAHMEIZUSTAND" (state of emergency)-Do feature films and documentaries on mental health reduce stigma and influence helpseeking attitudes? Psychiatry Res. 2014;220(3):1043-50.

84. Berridge BJ, Hall K, Dillon P, Hides L, Lubman DI. MAKINGtheLINK: a schoolbased health promotion programme to increase help-seeking for cannabis and mental health issues among adolescents. Early Interv Psychiatry. 2011; 5(1):81-8. https://doi.org/10.1111/j.1751-7893.2010.00252.x. 
85. Perry Y, Petrie K, Buckley H, Cavanagh L, Clarke D, Winslade M, Christensen $\mathrm{H}$. Effects of a classroom-based educational resource on adolescent menta health literacy: A cluster randomised controlled trial. J Adolesc. 2014;37(7): 1143-51. https://doi.org/10.1016/j.adolescence.2014.08.001.

86. Lubman DI, Berridge BJ, Blee F, Jorm AF, Wilson CJ, Allen NB, Wolfe R. A school-based health promotion programme to increase help-seeking for substance use and mental health problems: study protocol for a randomised controlled trial. BMC Trials. 2016;17(393). https://doi.org/10. 1186/s13063-016-1510-2.

87. Sharpe H, Patalay P, Vostanis P, Belsky J, Humphrey N, Wolpert M. Use, acceptability and impact of booklets designed to support mental health self-management and help seeking in schools: results of a large randomised controlled trial in England. Eur Child Adolesc Psychiatry. 2017;26(3):315-24. https://doi.org/10.1007/s00787-016-0889-3.

88. Casañas R, Arfuch VM, Castellví P, Gil JJ, Torres M, Pujol A, Castells G, Teixidó M, San-Emeterio MT, Sampietro HM, Caussa A, Alonso J, Lalucat-Jo L. EspaiJove.net- a school-based intervention programme to promote mental health and eradicate stigma in the adolescent population: study protocol for a cluster randomised controlled trial. BMC Public Health. 2018;18(1):939.

89. Kalafat J, Elias M. An evaluation of a school-based suicide awareness intervention. Suicide Life Threat Behav. 1994;24(3):224-33.

90. Aseltine R, DeMartino R. An outcome evaluation of the SOS suicide prevention program. Am J Public Health. 2004;94(3):446-51.

91. Aseltine R, James A, Schilling E, Glanovsky J. Evaluating the SOS suicide prevention program: a replication and extension. BMC Public Health. 2007; 7(161). https://doi.org/10.1186/1471-2458-7-161.

92. Freedenthal S. Adolescent help-seeking and the yellow ribbon suicide prevention program: an evaluation. Suicide Life Threat Behav. 2010;40(6): 628-39.

93. Schmidt S, Schimmelmann B. Mechanisms of change in psychotherapy for children and adolescents: current state, clinical implications, and methodological and conceptual recommendations for mediation analysis. Eur Child Adolesc Psychiatry. 2015;24:249-53.

94. Joyce A, Pauli-Myler T, Zazryn T, Batras D, Mayers K. Promotion help-seeking among adolescents and young adults through consideration of the adaptive functions of low mood: a pilot study. Int J Ment Health Promot. 2011;13(4):30-5.

95. Robinson J, Gook S, Yuen HP, Hughes A, Dodd S, Bapat S, Yung A. Depression education and identification in schools: an Australian-based study. Sch Ment Heal. 2010;2:13-22.

96. Ruble AE, Leon PJ, Gilley-Hensley L, Hess SG, Swartz KL. Depression knowledge in high school students: effectiveness of the adolescent depression awareness program. J Affect Disord. 2013;150(3):1025-30. https:// doi.org/10.1016/j.jad.2013.05.033.

97. Beaudry M, Swartz K, Miller L, Schweizer B, Glazer K, Willcox H. Effectiveness of the adolescent depression awareness program (ADAP) on depression literacy and mental health treatment. J Sch Health. 2019. https://doi.org/10. 1111/josh.12725.

98. Howard K, Griffiths K, McKetin R, Ma J. Can a brief biologically-based psychoeducational intervention reduce stigma and increase helpseeking intentions for depression in young people? A randomised controlled trial. J Child Adolesc Ment Health. 2018;30(1):27-39.

99. King K, Strunk C, Sorter MT. Preliminary effectiveness of surviving the teens ${ }^{\circledast}$ suicide prevention and depression awareness program on adolescents' Suicidality and self-efficacy in performing help-seeking behaviors. J School Health Am School Health Assoc. 2011;81:581-90.

100. Strunk CM, Sorter MT, Ossege J, King KA. Emotionally troubled teens' helpseeking behaviors: an evaluation of surviving the teens (R) suicide prevention and depression awareness program. J Sch Nurs. 2014;30(5):36675. https://doi.org/10.1177/1059840513511494.

101. Saporito, J. M. Reducing stigma toward seeking mental health treatment. Dissertation Abstracts International: Section B: The Sciences and Engineering; 2009;70(6-B):3794.

102. Hart LM, Mason RJ, Kelly CM, Cvetkovski S, Jorm AF. 'Teen mental health first aid': a description of the program and an initial evaluation. Int J Ment Heal Syst. 2016;10(3)

103. Rickwood D, Cavanagh S, Curtis L, Sakrouge R. Educating young people about mental health and mental illness: evaluating a school-based programme. Int J Ment Health Promot. 2004;6(4):23-32.

104. Young R, Hartman LI, Michel NM, Winter A, Flett GL, Goldberg JO. Reducing self-stigma toward help-seeking in high school youth: effects of classroom psycho-education about schizophrenia and mental illness. Schizophr Bull. 2013.

105. Calear AL, Banfield M, Batterham, PJ, et al. Silence is deadly: a clusterrandomised controlled trial of a mental health help-seeking intervention for young men. BMC Public Health. 2017;17:834. https://doi.org/10.1186/s12889017-4845-z.

106. Yang J, Cervera RL, Tye SJ, Ekker SC, Pierret C. Adolescent mental health education InSciEd out: a case study of an alternative middle school population. J Transl Med. 2018;16. https://doi.org/10.1186/s12967018-1459-x.

107. Rughani J. Adolescents' help-seeking for mental health problems: development and evaluation of a school-based intervention. Australia: School of Psychology, University of Wollongong; 2011. Doctor of Psychology (Clinical) Thesis.

108. Deane F, Wilson C, Russell N. Brief report: impact of classroom presentations about health and help-seeking on rural Australian adolescents' intentions to consult health care professionals. J Adolesc. 2007:30:695-9.

109. Wilson C, Deane F, Kellie E, Marshall E, Dalley A. Reducing adolescents' perceived barriers to treatment and increasing help-seeking intentions: effects of classroom presentations by general practitioners. J Youth Adolesc. 2008:37:1257-69.

110. Wilson CJ, Deane FP, Ciarrochi J, Rickwood D. Measuring help seeking intentions: properties of the general help seeking questionnaire. Can J Couns. 2006:39:15-28.

111. Nicholas J, Oliver K, Lee K, O'Brien M. Help-seeking behaviour and the internet: an investigation among Australian adolescents. Aust J Adv Ment Health. 2004;3(1):16-23. https://doi.org/10.5172/jamh.3.1.16.

112. Santor DA, Poulin C, LeBlanc JC, Kusumakar V. Facilitating help seeking behavior and referrals for mental health difficulties in school aged boys and girls: A school-based intervention. J Youth Adolesc. 2007;36(6):741-52. https://doi.org/10.1007/s10964-006-9092-z.

113. Santor DA, Poulin C, LeBlanc JC, Kusumakar V. Online health promotion, early identification of difficulties, and help seeking in young people. J Am Acad Child Adolesc Psychiatry. 2007;46(1):50-9. https://doi.org/10.1097/01. chi.0000242247.45915.ee.

114. Wiljer D, Abi-Jaoude A, Johnson A, Ferguson G, Sanches M, Levinson A Voineskos A. Enhancing self-efficacy for help-seeking among transition-aged youth in postsecondary settings with mental health and/or substance use concerns, using crowd-sourced online and mobile technologies: the thought spot protocol. JMIR Res Protoc. 2016;5(4). https://doi.org/10.2196/ resprot.6446.

115. Rowe SL, Patel K, French RS, Henderson C, Ougrin D, Slade M, Moran P. Web-based decision aid to assist help-seeking choices for young people who self-harm: outcomes from a randomized controlled feasibility trial. JMIR Mental Health. 2018;5(1). https://doi.org/10.2196/mental.8098.

116. Watkins L, O'Reilly J, Kuhn M, Gaverter C, Lancioni G, Sigafoos J, Lang R. A review of peer-mediated social interaction interventions for students with autism in inclusive settings. J Autism Dev Disord. 2014;45(4):1070-83.

117. Wyman P, Hendricks Brown C, LoMurray M, Schmeelk-Cone K, Petrova M, Yu Q, Walsh E, Tu X, Wang W. An outcome evaluation of the sources of strength suicide prevention program delivered by adolescent peer leaders in high schools. Am J Public Health. 2010;100(9):1653-61.

118. Calear AL, Brewer JL, Batterham PJ, Mackinnon A, Wyman PA, LoMurray M, Christensen $\mathrm{H}$. The sources of strength Australia project: study protocol for a cluster randomised controlled trial. Trials. 2016;17:349. https://doi.org/10. 1186/s13063-016-1475-1.

119. O'Reilly A, Barry J, Neary ML, Lane S, O'Keeffe L. An evaluation of participation in a schools-based youth mental health peer education training programme. Adv School Ment Health Promot. 2016;9(2):107-18. https://doi.org/10.1080/1754730x.2016.1154794.

120. Parikh S, Taubman V, Antoun DS, Cranford C, Foster J, Grambeau CE, Greden MJF. The Michigan peer-to-peer depression awareness program: schoolbased prevention to address depression among teens. Psychiatr Serv. 2018; 69(4):487-91.

121. Sawyer MG, Borojevic N, Ettridge KA, Spence SH, Sheffield J, Lynch J. Do help-seeking intentions during early adolescence vary for adolescents experiencing different levels of depressive symptoms? J Adolesc Health. 2012:50(3):236-42

122. Reardon T, Havery K, Baranowska M, O'Brien D, Smith L, Creswell C. What do parents perceive are the barriers and facilitators to accessing psychological treatment for mental health problems in children and adolescents? A 
systematic review of qualitative and quantitative studies. Eur Child Adolesc Psychiatry. 2017;26(6):623-47.

123. Kazdin A, Nock M. Delineating mechanisms of change in child and adolescent therapy: methodological issues and research recommendations. J Child Psychol Psychiatry. 2003;44(8):1116-29.

124. Asnel N. Children, youth and development. New York: Routledge, Perspectives on development; 2017.

125. Lee F, Heimer H, Giedd J, Edwards S, Sestan N, Weinberger D, Casey BJ. Adolescent mental health—opportunity and obligation. Emerging neuroscience offers hope for treatments. Sci Mag. 2014;346(6209):547-9.

126. Powell B, Proctor E, Glass J. A systematic review of strategies for implementing empirically supported mental health interventions. Res Soc Work Pract. 2014;24(2):192-212.

127. Owens J, Lyon A, Evangelista N, Warner C, Nadeem E, Spiel C, Wagner M. Implementation science in school mental health: key constructs in a developing research agenda. Sch Ment Heal. 2013. https://doi.org/10.1007/ s12310-013-9115-3.

128. Black AT, Seder RC, Kekahio W. Review of research on student nonenrollment and chronic absenteeism: a report for the Pacific region (REL 2015-054). [online]. Washington, DC: U.S. Department of Education, Institute of Education Sciences, National Center for Education Evaluation and Regional Assistance, Regional Educational Laboratory Pacific; 2014. Retrieved from http://ies.ed.gov/ncee/edlabs.

129. UNESCO. Fact sheet N. 48. One in five children, adolescents and youth is out of school. Montreal and Paris: United Nation Educational, Scientific and Cultural Organization; 2018. Available from: http://uis.unesco.org/sites/ default/files/documents/fs48-one-five-children-adolescents-youth-outschool-2018-en.pdf.

130. Zhang D, Wilson V, Katsiyannis A, Barrett D, Ju S, Wu J. Truancy offenders in the juvenile justice system: a multicohort study. Behav Disord. 2010;35(3): 229-42.

131. Chang H, Davis R. Mapping the early attendance gap: charting a course for student success. [Online]. Attendance Works \& Healthy Schools Campaign. 2015. Available from: http://www.attendanceworks.org/wp-content/ uploads/2017/05/Mapping-the-Early-Attendance-Gap_Final-4.pdf.

132. Rocha T, Graeff-Martins A, Kieling C, Rohde A. Provision of mental healthcare for children and adolescents: a worldwide view. Curr Opin Psychiatry. 2015;28(4):330-5.

133. Herrman H, Purcell R, Goldstone S, McGorry P. Improving mental health in young people. Psychiatr Danub. 2012;24(3):285-S290.

134. Newman L, Birleson P. Mental health planning for children and youth: is it developmentally appropriate? Aust Psychiatry. 2012;20(2):91-7.

135. Mokdad AH, Forouzanfar MH, Daoud F, et al. Global burden of diseases, injuries, and risk factors for young people's health during 1990-2013: a systematic analysis for the global burden of disease study 2013. Lancet. 2016;387(10036):2383-401.

136. Mayes L, Suchman N. Developmental pathways to substance abuse. In: Cicchetti D, Cohen D, editors. Developmental psychopathology: volume two. Developmental neuroscience, second edition. New York: Wiley; 2015.

\section{Publisher's Note}

Springer Nature remains neutral with regard to jurisdictional claims in published maps and institutional affiliations.

Ready to submit your research? Choose BMC and benefit from:

- fast, convenient online submission

- thorough peer review by experienced researchers in your field

- rapid publication on acceptance

- support for research data, including large and complex data types

- gold Open Access which fosters wider collaboration and increased citations

- maximum visibility for your research: over $100 \mathrm{M}$ website views per year

At BMC, research is always in progress.

Learn more biomedcentral.com/submissions 